\title{
WEAK MULTIPLICATIVE OPERATORS ON FUNCTION ALGEBRAS WITHOUT UNITS
}

\author{
THOMAS TONEV \\ The University of Montana, Missoula \\ Missoula, Montana 59812, USA \\ E-mail: tonevtv@mso.umt.edu
}

\begin{abstract}
For a function algebra $A$ let $\partial A$ be the Shilov boundary, $\delta A$ the Choquet boundary, $p(A)$ the set of $p$-points, and $|A|=\{|f|: f \in A\}$. Let $X$ and $Y$ be locally compact Hausdorff spaces and $A \subset C(X)$ and $B \subset C(Y)$ be dense subalgebras of function algebras without units, such that $X=\partial A, Y=\partial B$ and $p(A)=\delta A, p(B)=\delta B$. We show that if $\Phi:|A| \rightarrow|B|$ is an increasing bijection which is sup-norm-multiplicative, i.e. $\|\Phi(|f|) \Phi(|g|)\|=\|f g\|, f, g \in A$, then there is a homeomorphism $\psi: p(B) \rightarrow p(A)$ with respect to which $\Phi$ is a $\psi$-composition operator on $p(B)$, i.e. $(\Phi(|f|))(y)=|f(\psi(y))|, f \in A, y \in p(B)$. We show also that if $A \subset C(X)$ and $B \subset C(Y)$ are dense subalgebras of function algebras without units, such that $X=\partial A$, $Y=\partial B$ and $p(A)=\delta A, p(B)=\delta B$, and $T: A \rightarrow B$ is a sup-norm-multiplicative surjection, namely, $\|T f T g\|=\|f g\|, f, g \in A$, then $T$ is a $\psi$-composition operator in modulus on $p(B)$ for a homeomorphism $\psi: p(B) \rightarrow p(A)$, i.e. $|(T f)(y)|=|f(\psi(y))|, f \in A, y \in p(B)$. In particular, $T$ is multiplicative in modulus on $p(B)$, i.e. $|T(f g)|=|T f T g|, f, g \in A$. We prove also that if $A \subset C(X)$ is a dense subalgebra of a function algebra without unit, such that $X=\partial A$ and $p(A)=\delta A$, and if $T: A \rightarrow B$ is a weakly peripherally-multiplicative surjection onto a function algebra $B$ without unit, i.e. $\sigma_{\pi}(T f T g) \cap \sigma_{\pi}(f g) \neq \emptyset, f, g \in A$, and preserves the peripheral spectra of algebra elements, i.e. $\sigma_{\pi}(T f)=\sigma_{\pi}(f), f \in A$, then $T$ is a bijective $\psi$-composition operator on $p(B)$, i.e. $(T f)(y)=f(\psi(y)), f \in A, y \in p(B)$, for a homeomorphism $\psi: p(B) \rightarrow p(A)$. In this case $A$ is necessarily a function algebra and $T$ is an algebra isomorphism. As a consequence, a multiplicative operator $T$ from a dense subalgebra $A \subset C(X)$ of a function algebra $B$ without unit, such that $X=\partial A$ and $p(A)=\delta A$, onto a function algebra without unit $B$ is a sup-norm isometric algebra isomorphism if and only if $T$ is weakly peripherallymultiplicative and preserves the peripheral spectra of algebra elements. The results extend to function algebras without units a series of previous results for algebra isomorphisms.
\end{abstract}

2010 Mathematics Subject Classification: Primary 46J10, 46J20; Secondary 46H40.

Key words and phrases: semisimple commutative Banach algebra, function algebra, uniform algebra, peaking function, peak set, strong boundary point, Choquet boundary, peripheral spectrum, composition operator, algebra isomorphism.

The paper is in final form and no version of it will be published elsewhere. 
1. Introduction. The search for universal criteria for operators between two Banach algebras to be linear and multiplicative, i.e. algebra isomorphisms, has been intensive for years. The initial findings were related to operators a priori known to be linear. In 2001 Molnár [9, 10] introduced spectral multiplicative operators, for which $\sigma(T f T g)=$ $\sigma(f g), f, g \in C(X)$, where $\sigma(f)$ is the spectrum of $f$, and found sufficient conditions for a spectrally-multiplicative self-map of $C(X)$ on a first-countable compact space $X$ to be an algebra isomorphism. In [11] Rao and Roy extended this result for a self-map of a uniform algebra, and in [12] for a self-map of a function algebra without unit. In [2] it was proven for surjections between distinct uniform algebras, in [3] for surjections between semisimple commutative Banach algebras with units, and in [4] between completely regular commutative Banach algebras without units. Norm-multiplicative operators, for which $\|T f T g\|=\|f g\|, f, g \in A$, were introduced in [7, where sufficient conditions for a norm-multiplicative operator between uniform algebras to be a composition operator in modulus were obtained. The peripheral spectrum $\sigma_{\pi}(f)=\{f(x):|f(x)|=\|f\|\}$, peripherally-multiplicative operators, for which $\sigma_{\pi}(T f T g)=\sigma_{\pi}(f g), f, g \in A$, and weakly peripherally-multiplicative operators, for which $\sigma_{\pi}(T f T g) \cap \sigma_{\pi}(f g) \neq \emptyset$, were introduced in [1], 8] and [7] respectively, where sufficient conditions for such operators to be algebra isomorphisms were established. In the case of non-unital Lipschitz algebras similar results were obtained in [6].

In this paper we extend the above results to sup-norm-multiplicative and weakly peripherally-multiplicative operators between algebras of functions without units, the uniform closures of which are function algebras. For a function algebra $A$ denote by $\partial A$ the Shilov boundary, by $\delta A$ the Choquet boundary, by $p(A)$ the set of $p$-points, and by $|A|=\{|f|: f \in A\}$ the modulus of $A$. Let $X$ and $Y$ be locally compact Hausdorff spaces and $A \subset C(X)$ and $B \subset C(Y)$ be algebras of functions without units, the uniform closures of which are function algebras without units, such that $X=\partial A, Y=\partial B$ and $p(A)=\delta A, p(B)=\delta B$.

The structure of the paper is as follows. In Section 2 we show that if $\Phi:|A| \rightarrow|B|$ is an increasing bijection which is sup-norm-multiplicative, i.e. $\|\Phi(|f|) \Phi(|g|)\|=\|f g\|$ for all $f, g \in A$, then there exists a homeomorphism $\psi: p(B) \rightarrow p(A)$ such that $\Phi$ is a $\psi$-composition operator on $p(B)$, i.e. $(\Phi(|f|))(y)=|f(\psi(y))|$ for all $f \in A$ and $y \in p(B)$. In particular, $\Phi$ is multiplicative, i.e. $(\Phi(|f g|)=\Phi(|f|) \Phi(|g|)$ for all $f, g \in A$. As a consequence, if $T: A \rightarrow B$ is a sup-norm-multiplicative surjection, namely, $\|T f T g\|=$ $\|f g\|$ (or, $\mathfrak{r}(T f T g)=\mathfrak{r}(f g)$, where $\mathfrak{r}(\cdot)$ is the spectral radius), for all $f, g \in A$, then $T$ is a $\psi$-composition operator in modulus on $p(B)$ for a homeomorphism $\psi: p(B) \rightarrow p(A)$, i.e. $|(T f)(y)|=|f(\psi(y))|$ for all $f \in A$ and $y \in p(B)$. In particular, $T$ is multiplicative in modulus on $p(B)$, i.e. $|T(f g)|=|T f T g|$ for all $f, g \in A$. In Section 3 we prove a strong version of the classical Bishop's Lemma for function algebras without units, and show that if $A \subset C(X)$ is a dense subalgebra of a function algebra without unit, such that $X=\partial A$ and $p(A)=\delta A$, and $T: A \rightarrow B$ is a weakly peripherally-multiplicative surjection onto a function algebra $B$ without unit, i.e. $\sigma_{\pi}(T f T g) \cap \sigma_{\pi}(f g) \neq \emptyset$ for all $f, g \in A$, and preserves the peripheral spectra of algebra elements, i.e. $\sigma_{\pi}(T f)=\sigma_{\pi}(f), f \in A$, then $T$ is a bijective $\psi$-composition operator on $p(B)$, i.e. $(T f)(y)=f(\psi(y))$ for all $f \in A$ and $y \in p(B)$, with respect to a homeomorphism $\psi: p(B) \rightarrow p(A)$. In this case $A$ is 
necessarily a function algebra and $T$ is an algebra isomorphism. As a consequence, an operator $T$ from a dense subalgebra $A \subset C(X)$ of a function algebra without unit, such that $X=\partial A$ and $p(A)=\delta A$ onto a function algebra $B$ without unit is a sup-norm isometric algebra isomorphism if and only if $T$ is weakly peripherally-multiplicative and preserves the peripheral spectra of algebra elements. In particular, any multiplicative surjection $T$ from a dense subalgebra $A \subset C(X)$ of a function algebra $B$ without unit, such that $X=\partial A$ and $p(A)=\delta A$, onto a function algebra without unit $B$ is a sup-norm isometric algebra isomorphism if and only if $T$ preserves the peripheral spectra of algebra elements.

The results extend for function algebras without units the main results in a series of recent papers (e.g. [1, 2, 17, 8, 10, 11, 12]) for algebra isomorphisms.

\section{Norm-multiplicative operators on the modulus of an algebra. Let $A$ be} a semisimple commutative Banach algebra, not necessarily unital, with maximal ideal space $\mathcal{M}_{A}$, and Shilov boundary $\partial A$. We identify $A$ with $\widehat{A}$ and $a \in A$ with $\widehat{a}$, the corresponding Gelfand transforms. Under this agreement, $\left.A \cong A\right|_{\partial A}$. By $\|f\|$ we will denote the spectral norm $\mathfrak{r}(f)$ of $f \in A$. We assume that any algebra $A$ in this paper is without unit and is supported on its Shilov boundary $\partial A$, i.e. $A \subset C(\partial A)$. Let $X$ be a locally compact Hausdorff space. A function algebra on $X$ is a semisimple commutative Banach algebra $A \subset C(X)$ which is closed under the sup-norm $\|f\|=\sup _{x \in X}|f(x)|$. Denote by $\delta A$ the Choquet boundary of $A$. Note that the uniform closure $\bar{A}$ of any semisimple commutative Banach algebra $A \subset C(X)$ is a function algebra. If $A$ is unital, then its Shilov boundary $\partial A$ and maximal ideal space $\mathcal{M}_{A}$ are compact spaces. If $A$ is not unital, then $\partial A$ and $\mathcal{M}_{A}$ are locally compact spaces and $\widehat{A} \subset C_{0}\left(\mathcal{M}_{A}\right)$, the space of continuous functions on $\mathcal{M}_{A}$ that vanish at infinity. Note that if $V$ is an open set of $X=\partial A$, then $\sup _{x \in X \backslash V}|f(x)|$ is attained in $X \backslash V$ for any $f \in A$.

Let $X$ be a locally compact Hausdorff space and $B$ is a subset of a function algebra $A \subset C(X)$ with $\partial A=X$. For a $f \in B$ denote by $E(f)=E(|f|)=\{x \in X:|f(x)|=\|f\|\}$ the maximum modulus set of $f$. Note that for every $f \in B$ and $0<c<\|f\|$ the set $\{x \in X:|f(x)| \geq c\}$ is compact, and so is the set $E(f)$. An $h \in B$ with $\|h\|=1$ and $|h(x)|<1$ whenever $h(x) \neq 1$ is called a peaking function of $B$. If $h$ is a peaking function of $B$ and $E \subset E(h)$ we say that $h$ peaks on $E$. The set of all peaking functions of $B$ will be denoted by $\mathcal{P}(B)$. Denote $\mathcal{S}(B)=\{f \in B:\|f\|=1\}$ and, given an $E \subset X, \mathcal{F}_{E}(B)=$ $\{f \in \mathcal{S}(B):|f(x)|=1$ for all $x \in E\}$. The set of all peaking functions of $B$ that peak on $E$ will be denoted by $\mathcal{P}_{E}(B)$. If $h \in \mathcal{P}_{E}(B)$, then, clearly, $E$ is in the maximum modulus set $E(h)$ of $h$, so $h \in \mathcal{F}_{E}(B)$, thus $\mathcal{P}_{E}(B) \subset \mathcal{F}_{E}(B)$. A set $E \subset X$ is called a peak set for $B$ if $E$ is the maximum modulus set $E(h)=E(|h|)=\{x \in X:|h(x)|=1\}=h^{-1}\{1\}$ of a peaking function $h \in B$. A non-empty subset $E$ of $X$ is called a $p$-set for $B$ if for every open set $V \supset E$ there is a peaking function $h \in \mathcal{P}_{E}(B)$ so that $E(h) \subset V$. One can readily see that $E$ is a $p$-set for $B$ if and only if $E$ is an intersection of a family of peak sets. A point $x \in X$ is called a p-point, or a strong boundary point for $B$ if $\{x\}$ is a $p$-set, i.e. if for every neighborhood $V$ of $x$ there is a peaking function $h$ of $B$ so that $h(x)=1$ and $E(h) \subset V$. The set of all $p$-points for $B$ will be denoted by $p(B)$. If $A$ 
is a function algebra then the set $p(A)$ of peak points of $A$ is a boundary, namely, the Choquet boundary $\delta A$ (see e.g. [12]). Note that if $A$ is a dense subalgebra of a function algebra equipped with the sup-norm, then $\delta A=\delta \bar{A}$. Clearly, for every neighborhood $U$ of a peak set $E \subset X$ for $B$ there is an $h \in \mathcal{F}_{E}(B)$ such that $E(h) \subset U$.

Every $p$-point of a semisimple commutative Banach algebra $A$ belongs to the Choquet boundary $\delta A$. It may happen that $A$ does not have $p$-points at all. The opposite case, when $p(A)=\delta A$ happens, for instance, if $A$ is a uniform algebra, or, a function algebra, or, a completely regular, not necessarily unital, Banach function algebra on $\mathcal{M}_{A}$, in which case $p(A)=\mathcal{M}_{A}$. In what follows, $X$ denotes a locally compact Hausdorff space.

LEMma 2.1. Let $A \subset C(X)$ be a subalgebra of a function algebra without unit, such that $X=\partial A$. If $h \in \mathcal{P}(A)$ and $V \subset X$ is an open set containing $E(h)$, then $\sup _{X \backslash V}|h(x)|<1$.

Proof. Suppose on the contrary that $\sup _{X \backslash V}|h(x)|=1$. Since $h$ is continuous and $\lim _{x \rightarrow \infty} \widehat{h}(x)=0$, by the remark from the above there is an $x_{0} \in X \backslash V$ with $\left|h\left(x_{0}\right)\right|=$ $\sup _{X \backslash V}|h(x)|=1$, i.e. $x_{0} \in E(h)$, which is impossible.

LEMma 2.2. Let $A \subset C(X)$ be a subalgebra of a function algebra without unit, such that $X=\partial A$, and let $E \subset X$ be a nonempty p-set for $A$. Then $\max _{x \in E}|f(x)|=\inf _{h \in \mathcal{F}_{E}(A)}\|f h\|$ for any $f \in A$. In particular, if $x_{0}$ is a p-point for $A$ then $\left|f\left(x_{0}\right)\right|=\inf _{h \in \mathcal{F}_{x_{0}}(A)}\|f h\|$.

Proof. Let $f \in A$ and $\varepsilon>0$. Consider the open set $V=\left\{x \in X:|f(x)|<\max _{\xi \in E}|f(\xi)|+\varepsilon\right\}$, which clearly contains $E$. Let $k \in \mathcal{P}_{E}(A)$ be such that $E(k) \subset V$. By Lemma 2.1. $\sup _{X \backslash V}|k(x)|<1$. Hence there is a large enough power $h$ of $k$ such that $|f(x) h(x)| \leq$ $\max _{\xi \in E}|f(\xi)|+\varepsilon$ for all $x \in X \backslash V$. Since $|f(x) h(x)| \leq|f(x)|<\max _{\xi \in E}|f(\xi)|+\varepsilon$ for all $x \in V$, we deduce that $\|f h\| \leq \max _{\xi \in E}|f(\xi)|+\varepsilon$. Therefore,

$$
\max _{\xi \in E}|f(\xi)|=\max _{\xi \in E}|f(\xi) h(\xi)| \leq\|f h\|<\max _{\xi \in E}|f(\xi)|+\varepsilon .
$$

It follows that $\max _{\xi \in E}|f(\xi)|=\inf _{h \in \mathcal{F}_{E}(B)}\|f h\|$, as claimed.

LEMma 2.3. Let $A \subset C(X)$ be a dense subalgebra of a function algebra without unit, such that $X=\partial A$ and $p(A)=\delta A$. Then

(a) For any $f \in A$ the set $E(f)$ is a disjoint union of peak sets for $A$.

(b) Every set of type $E=\bigcap_{\alpha} E\left(f_{\alpha}\right), f_{\alpha} \in \mathcal{S}(B)$, meets $p(A)$, i.e $E \cap p(A) \neq \emptyset$.

Proof. If $f \in \mathcal{S}(A)$ then, clearly, $E(f)=\bigcup\left\{f^{-1}\{\lambda\}:|\lambda|=1, \lambda \in \operatorname{Ran}(f)\right\}$. Fix a $\lambda \in$ $\operatorname{Ran}(f)$ with $|\lambda|=1$. Consider the function

$$
g=\frac{1}{2 \lambda} \cdot\left(\frac{f^{2}}{\lambda}+f\right) \in A .
$$

For any $x \in f^{-1}\{\lambda\}$ we have $g(x)=1$, while

$$
|g(x)|=\frac{|f(x)|}{2}\left|\frac{f(x)}{\lambda}+1\right| \leq \frac{1}{2}\left|\frac{f(x)}{\lambda}+1\right|<1 \quad \text { whenever } x \notin f^{-1}\{\lambda\} .
$$

Therefore, $g \in \mathcal{P}(A)$ and $E(g)=f^{-1}(\lambda)$. Consequently, $f^{-1}(\lambda)$ is a peak set for $A$. This proves $(a)$. 
Let $E=\bigcap_{\alpha} E\left(f_{\alpha}\right) \neq \emptyset, f_{\alpha} \in \mathcal{S}(B)$, and let $x \in E$. Then $x$ belongs to a peak set $P_{\alpha} \subset E_{\alpha}$ of $A$ for every $\alpha$. Consequently, $\bigcap_{\alpha} P_{\alpha} \subset E$ is a $p$-set for $A$ and also for $\bar{A}$. Therefore, the set $\bigcap_{\alpha} P_{\alpha}$ meets $\delta \bar{A}=\delta A=p(A)$ (cf. [12]), and so does $E$.

Let $X$ and $Y$ be locally compact Hausdorff spaces, $A \subset C(X)$ and $B \subset C(Y)$ are dense subalgebras of function algebras on $X=\partial A$ and $Y=\partial B$ with $p(A)=\delta A$ and $p(B)=\delta B$. For a set $S \subset C(X)$ denote by $|S|=\{|f|: f \in S\}$ the modulus of $S$. An operator $\Phi:|A| \rightarrow|B|$ is increasing if for every $f, g \in A$ the inequality $|f| \leq|g|$ holds on $X$ if and only if the inequality $\Phi(|f|) \leq \Phi(|g|)$ holds on $Y$ and a $\psi$-composition operator on $p(B)$, if there is a map $\psi: p(B) \rightarrow X$ such that $(\Phi(|f|))(y)=|f(\psi(y))|$ for all $f \in A$ and $y \in p(B)$.

Proposition 2.4. Let $X$ and $Y$ be locally compact Hausdorff spaces, $A \subset C(X)$ and $B \subset C(Y)$ be dense subalgebras of function algebras without units, such that $X=\partial A$, $Y=\partial B$ and $p(A)=\delta A, p(B)=\delta B$, and let $\Phi:|A| \rightarrow|B|$ be an increasing bijection. If $\Phi$ is sup-norm-multiplicative, i.e.

$$
\|\Phi(|f|) \Phi(|g|)\|=\|f g\|
$$

for all $f, g \in A$, then there exists a homeomorphism $\psi: p(B) \rightarrow p(A)$ with respect to which $\Phi$ is a $\psi$-composition operator on $p(B)$, i.e. $(\Phi(|f|))(y)=|f(\psi(y))|$ for all $f \in A$ and $y \in p(B)$. In particular, $\Phi$ is multiplicative, i.e. $\Phi(|f g|)=\Phi(|f|) \Phi(|g|)$ for all $f, g \in A$.

Proposition 2.4 holds, say, for semisimple commutative Banach algebras $A, B$ on $X=$ $\partial A$ and $Y=\partial B$ with $p(A)=\delta A$ and $p(B)=\delta B$. Note that equality (1) implies automatically that $\|\Phi(|f|)\|=\|f\|$ for all $f \in A$.

For the proof we will use, with necessary adjustments, the technique developed in [11] and widely used later in $[2,4,6,6,17,12$. First we establish several preliminary lemmas, in all of which $A, B$ and $\Phi$ are as in Proposition 2.4

LeMma 2.5. For any $y \in p(B)$ the set

$$
E_{y}=\bigcap_{\substack{f \in A \\ \Phi(|f|) \in\left|\mathcal{F}_{y}(B)\right|}} E(f)
$$

is nonempty and $E_{y} \cap p(A) \neq \emptyset$.

Proof. We claim that the family $\left\{E(f): f \in A, \Phi(|f|) \in\left|\mathcal{F}_{y}(B)\right|\right\}$ has the finite intersection property. Let $f_{1}, f_{2}, \ldots, f_{n} \in A$ be such that $\Phi\left(\left|f_{k}\right|\right) \in\left|\mathcal{F}_{y}(B)\right|$ for $k=1,2, \ldots, n$. Then there is an $f \in A$ so that $\Phi(|f|)=\Phi\left(\left|f_{1}\right|\right) \Phi\left(\left|f_{2}\right|\right) \cdots \Phi\left(\left|f_{n}\right|\right) \in\left|\mathcal{F}_{y}(B)\right|$. Since $\Phi(|f|) \leq \Phi\left(\left|f_{k}\right|\right)$ on $Y$ and $\Phi$ is increasing, $|f(\xi)| \leq\left|f_{k}(\xi)\right|$ for all $\xi \in X$ and $k=1,2, \ldots, n$. Note that $\|f\|=\|\Phi(|f|)\|=1$ and also $\left\|f_{k}\right\|=\left\|\Phi\left(\left|f_{k}\right|\right)\right\|=1$. Hence for every $x \in X$ with $|f(x)|=1$ we must have $\left|f_{k}(x)\right|=1$ for all $k=1,2, \ldots, n$. Thus $E(f) \subset E\left(f_{k}\right)$ for every $k=1, \ldots, n$, and therefore, $E(f) \subset \bigcap_{k=1}^{n} E\left(f_{k}\right)$. Consequently, the family $\left\{E(f): f \in A, \Phi(|f|) \in\left|\mathcal{F}_{y}(B)\right|\right\}$ has the finite intersection property, as claimed. Since its elements, $E(f)$, are compact subsets of $X$, it must have nonempty intersection. According to Lemma $2.3(b), E_{y} \cap p(A) \neq \emptyset$. 
LEMMA 2.6. If $y \in p(B)$ and $x \in E_{y} \cap p(A)$, then

$$
\Phi\left(\left|\mathcal{F}_{x}(A)\right|\right) \subset\left|\mathcal{F}_{y}(B)\right|
$$

Proof. Fix an $h \in\left|\mathcal{F}_{x}(A)\right|$ and let $k=\Phi(h)$. To prove that $\Phi(h) \in\left|\mathcal{F}_{y}(B)\right|$, it suffices to show that $k(y)=1$. Take an open neighborhood $V$ of $y$ in $Y$ and a $q \in \mathcal{F}_{y}(B)$ with $E(q) \subset V$. If $p=\Phi^{-1}(|q|)$ then $\|p\|=\|q\|=1$, thus

$$
E_{y} \cap p(A) \subset E_{y}=\bigcap_{\substack{f \in A \\ \Phi(|f|) \in\left|\mathcal{F}_{y}(B)\right|}} E(f) \subset E(p),
$$

since $\Phi(p)=|q| \in\left|\mathcal{F}_{y}(B)\right|$. Therefore, $p \in\left|\mathcal{F}_{x}(A)\right|$, since, by the hypotheses, $x \in E_{y} \cap$ $p(A)$. Equality (1) implies $1=h(x) p(x)=\|h p\|=\|k q\| \leq\|k\|\|q\|=1$. Consequently, $\|k q\|=1$ and there must be a $y_{V} \in p(B)$ such that $k\left(y_{V}\right)=\left|q\left(y_{V}\right)\right|=1$. Therefore, $y_{V} \in E(q) \subset V$. Since any neighborhood $V$ of $y$ contains a point $y_{V}$ with $k\left(y_{V}\right)=1$, the continuity of $k$ implies that $k(y)=1$. Hence, $k \in\left|\mathcal{F}_{y}(B)\right|$ and therefore, $\Phi\left(\left|\mathcal{F}_{x}(A)\right|\right) \subset$ $\left|\mathcal{F}_{y}(B)\right|$, as claimed.

Lemma 2.7. For any $y \in p(B)$ the set $E_{y} \cap p(A)$ is a singleton.

Proof. Let $y \in p(B), x \in E_{y} \cap p(A)$ and $z \in\left(E_{y} \cap p(A)\right) \backslash\{x\}$. Since $|A|$ separates the points of $p(A)$, there is a function $h \in \mathcal{F}_{x}(A)$ such that $|h(z)|<|h(x)|=1$. Lemma 2.6 implies that $\Phi(|h|) \in\left|\mathcal{F}_{y}(B)\right|$, and therefore, $E_{y} \cap p(A) \subset E(h)$, by (2). Hence, $|h| \equiv 1$ on $E_{y} \cap p(A)$, which is impossible since $|h(z)|<1$. Therefore, the set $E_{y} \cap p(A)$ contains no points other than $x$.

Given an $y \in p(B)$ we denote the single element of the set $E_{y} \cap p(A)$ by $\psi(y)$, i.e., $\{\psi(y)\}=E_{y} \cap p(A)$. Hence there arises a map $\psi: y \leadsto \psi(y)$ from $p(B)$ to $p(A)$, and (3) can be rewritten as

$$
\Phi\left(\left|\mathcal{F}_{\psi(y)}(A)\right|\right) \subset\left|\mathcal{F}_{y}(B)\right| .
$$

If $h \in \mathcal{F}_{\psi(y)}(A)$ then $k=\Phi(|h|) \in\left|\mathcal{F}_{y}(B)\right|$ by (4). Therefore, $|h(\psi(y))|=1=(\Phi(|h|))(y)$, and hence

$$
(\Phi(|h|))(y)=|h(\psi(y))|
$$

for any $h \in \mathcal{F}_{\psi(y)}(A)$.

Corollary 2.8. For any $y \in p(B), \Phi^{-1}\left(\left|\mathcal{F}_{y}(B)\right|\right)=\left|\mathcal{F}_{\psi(y)}(A)\right|$.

Proof. Let $y \in p(B), k \in \mathcal{F}_{y}(B)$ and $h=\Phi^{-1}(|k|)$. Then $\Phi(h)=|k| \in\left|\mathcal{F}_{y}(B)\right|$, and therefore, $\psi(y) \in E(h)$ by $(2)$. Hence, $h(\psi(y))=1=|k(y)|$. Since $h(\psi(y))=\|h\|$, we have that $h \in\left|\mathcal{F}_{\psi(y)}(A)\right|$. Therefore, $\Phi^{-1}\left(\left|\mathcal{F}_{y}(B)\right|\right) \subset\left|\mathcal{F}_{\psi(y)}(A)\right|$. The opposite inclusion follows from (4).

What Proposition 2.4 claims is that the equality (5) holds actually for every $h \in A$ and all $y \in p(B)$.

Proof of Proposition 2.4. Let $f \in A$ and $y \in p(B)$. Lemma 2.2 Corollary 2.8, and (1) imply 


$$
\begin{aligned}
|(\Phi(|f|))(y)| & =\inf _{k \in \mathcal{F}_{y}(B)}\|(\Phi(|f|)) \cdot|k|\|=\inf _{h \in \mathcal{F}_{\psi(y)}(A)}\|\Phi(|f|) \Phi(|h|)\| \\
& =\inf _{h \in \mathcal{F}_{\psi(y)}(A)}\|f h\|=|f(\psi(y))| .
\end{aligned}
$$

Therefore, $\Phi$ is a $\psi$-composition operator on $p(B)$. Let $y \in p(B)$ and $0<c<1$. Choose an open set $U$ of $\psi(y)$ in $X$ and a function $h \in \mathcal{P}_{\psi(y)}(A)$ such that $E(h) \subset U$ and $|h(x)|<c$ for all $x \in X \backslash U$. Then $k=\Phi(|h|) \in\left|\mathcal{F}_{y}(B)\right|$ and, according to (5), $k(y)=$ $|h(\psi(y))|=1>c$. Therefore, the open set $W=\{\eta \in p(B): k(\eta)>c\}$ contains $y$. The first part of the proof shows that $|h(\psi(\eta))|=k(\eta)>c$ for every $\eta \in W$, which implies $\psi(\eta) \in U$, since $|h(\xi)|<c$ on $X \backslash U$. Hence $\psi(W) \subset U$. Consequently, $\psi$ is continuous. Since $\Phi$ is bijective, then its inverse, $\Phi^{-1}:|B| \rightarrow|A|$, is surjective and, by symmetry, satisfies the hypotheses of Proposition 2.4. By the first two parts of the proof there is a continuous mapping $\phi: p(A) \rightarrow p(B)$ so that $\left(\Phi^{-1}(|g|)\right)(x)=|g(\phi(x))|$ for every $g \in B$ and all $x \in p(A)$. It is easy to see that the mappings $\phi$ and $\psi$ are inverses to each other, and therefore are homeomorphisms. For any $f, g \in A$ and $y \in p(B)$ we have $(\Phi(|f g|))(y)=|(f g)(\psi(y))|=|f(\psi(y))||g(\psi(y))|=(\Phi(|f|))(y)(\Phi(|g|))(y)$, i.e. $\Phi$ is multiplicative.

Norm-multiplicative operators were introduced in [7], where a preliminary version of the next theorem was proven for a priory non-surjective operators between uniform algebras, that preserve the peaking functions. For surjective operators between uniform algebras its version was proven in [2], and between completely regular Banach function algebras - in [4].

TheOREM 2.9. Let $X$ and $Y$ be locally compact Hausdorff spaces and $A \subset C(X)$ and $B \subset C(Y)$ be dense subalgebras of function algebras without units, such that $X=\partial A, Y=$ $\partial B$ and $p(A)=\delta A, p(B)=\delta B$. If a surjection $T: A \rightarrow B$ is sup-norm-multiplicative, namely, $\|T f T g\|=\|f g\|$ for all $f, g \in A($ or, $\mathfrak{r}(T f T g)=\mathfrak{r}(f g)$, where $\mathfrak{r}(\cdot)$ is the spectral radius), then $T$ is a $\psi$-composition operator in modulus on $p(B)$ for a homeomorphism $\psi: p(B) \rightarrow p(A)$, i.e.

$$
|(T f)(y)|=|f(\psi(y))|
$$

for all $f \in A$ and $y \in p(B)$. In particular, $T$ is multiplicative in modulus on $p(B)$, i.e. $|T(f g)|=|T f T g|$ for all $f, g \in A$.

Proof. The sup-norm-multiplicativity of $T$ implies that $T$ preserves the sup-norms. Let $f, g \in A$ be such that $|T f| \leq|T g|$ on $Y$. For any $h \in A$ we have $\|f h\|=\|T f T h\| \leq$ $\|T g T h\|=\|g h\|$ by the sup-norm-multiplicativity. Lemma 2.2 implies that $|f| \leq|g|$ on $X$. If $|f| \leq|g|$ on $X$, then for every $k \in B$ we have $\|T f \cdot k\|=\|T f T h\|=\|f h\| \leq\|g h\|=$ $\|T g T h\|=\|T g \cdot k\|$, where $h \in T^{-1}(k)$. Therefore, $|T f| \leq|T g|$ on $Y$ by Lemma 2.2 . Consequently, $|f| \leq|g|$ holds on $X$ if and only if $|T f| \leq|T g|$ holds on $Y$. Therefore, $|T f| \equiv|T g|$ on $Y$ if and only if $|f| \equiv|g|$ on $X$. Hence the operator $\Phi:|A| \rightarrow|B|$ defined by $\Phi(|f|)=|(T f)|, f \in A$, is well defined. If $\Phi(|f|)=\Phi(|g|)$, then $|T f|=|T g|$ and therefore, $|f|=|g|$. Hence, $\Phi$ is injective and therefore, bijective, and also increasing. Consequently, $\Phi$ satisfies the hypotheses of Proposition 2.4 and therefore, it is a $\psi$-composition operator on $p(B)$ with respect to a homeomorphism $\psi: p(B) \rightarrow p(A)$, i.e. $(\Phi(|f|))(y)=|f(\psi(y))|$ 
for all $f \in A$ and $y \in p(B)$. Therefore, $|(T f)(y)|=(\Phi(|f|))(y)=|f(\psi(y))|$, i.e. $T$ is a $\psi$-composition operator in modulus on $p(B)$. The multiplicativity in modulus of $T$ follows from the multiplicativity of $\Phi$.

In particular, Theorem 2.9 holds for function algebras without units and, more generally, for semisimple commutative Banach algebras $A, B$ on $X=\partial A$ and $Y=\partial B$ with $p(A)=\delta A$ and $p(B)=\delta B$. A similar result is obtained in [5].

3. Weakly peripherally-multiplicative operators. The following proposition is a strong version for function algebras without units of the classical lemma of E. Bishop.

Proposition 3.1 (Strong Multiplicative Bishop's Lemma). Let $X$ be a locally compact Hausdorff space and $A \subset C(X)$ be a function algebra without unit on $X=\partial A$. If $f \in A$ and $x_{0} \in X$ is a p-point of $A$ with $f\left(x_{0}\right) \neq 0$, then there exists a peaking function $h_{0} \in \mathcal{P}_{x_{0}}(A)$ such that

$$
\sigma_{\pi}\left(f h_{0}\right)=\left\{f\left(x_{0}\right)\right\} .
$$

If, moreover, $E$ is a peak set of $A$ which contains $x_{0}$, then $h_{0}$ can be chosen so that $E\left(f h_{0}\right)=E\left(h_{0}\right) \subset E$.

We prove Proposition 3.1 by a technique similar to the one in [13].

Proof. Without loss of generality we can assume that $f\left(x_{0}\right)=1$. For every $n \in \mathbb{N}$ define the open set $U_{n}=\left\{x \in X:|f(x)-1|<1 / 2^{n+1}\right\}$. Clearly, $x_{0} \in U_{n} \subset U_{n-1}$ and $U_{n}$ is open in $X$ for all $n>1$. For every $n \in \mathbb{N}$ we choose a peaking function $k_{n} \in \mathcal{P}_{x_{0}}(A)$ such that $E\left(k_{n}\right) \subset U_{n}$. Let $h_{n}$ be a large enough power of $k_{n}$ such that $\left|h_{n}(x)\right|<1 /\left(2^{n}\|f\|\right)$ on $X \backslash U_{n}$. We claim that $\sigma_{\pi}\left(f h_{0}\right)=\{1\}$ holds for the function $h_{0}=\sum_{n=1}^{\infty} h_{n} / 2^{n}$. Clearly, $h_{0} \in \mathcal{P}_{x_{0}}(A)$. Note that $E\left(h_{0}\right) \subset \bigcap_{n=1}^{\infty} E\left(h_{n}\right) \subset \bigcap_{n=1}^{\infty} U_{n}$. In fact, $\bigcap_{n=1}^{\infty} U_{n}=f^{-1}\{1\}$. Indeed, if $x \in f^{-1}\{1\}$ then, clearly, $x \in \bigcap_{n=1}^{\infty} U_{n}$; if $x \in \bigcap_{n=1}^{\infty} U_{n}$, then $|f(x)-1|<1 / 2^{n+1}$ for every $n \in \mathbb{N}$, so $f(x)=1$, i.e. $x \in f^{-1}\{1\}$. Consequently, $E\left(h_{0}\right) \subset \bigcap_{n=1}^{\infty} U_{n}=f^{-1}\{1\}$. If $x \in E\left(h_{0}\right)$, then $f(x) h_{0}(x)=1$, while $\left|f(x) h_{0}(x)\right|<1$ when $x \in f^{-1}\{1\} \backslash E(h)$. Let $x \notin f^{-1}\{1\}$. If also $x \in X \backslash U_{1}$, then $x \in X \backslash U_{n}$ for all $n$, and therefore, $\left|h_{n}(x)\right|<$ $1 /\left(2^{n}\|f\|\right)$ for all $n$. Thus,

$$
\left|h_{0}(x)\right|<\sum_{n=1}^{\infty} \frac{1}{4^{n}\|f\|}<\frac{1}{\|f\|},
$$

and therefore, $\left|f(x) h_{0}(x)\right|<|f(x)| /\|f\| \leq 1$. If $x \in U_{n-1} \backslash U_{n}$ for some $n>1$, then $x \in U_{1}, U_{2}, \ldots, U_{n-1}$ and $x \in X \backslash U_{i}$ for all $i \geq n$. Consequently, $\left|h_{i}(x)\right|<1 /\left(2^{i}\|f\|\right)$ for all $i \geq n$. Since $x \in U_{n-1},|f(x)-1|<1 / 2^{n}$, and hence

$$
\begin{aligned}
\left|f(x) h_{0}(x)\right| & \leq(1+|f(x)-1|)\left|h_{0}(x)\right|<\left(1+\frac{1}{2^{n}}\right)\left(\sum_{i=1}^{n-1} \frac{\left|h_{i}(x)\right|}{2^{i}}+\sum_{i=n}^{\infty} \frac{\left|h_{i}(x)\right|}{2^{i}}\right) \\
& =\left(1+\frac{1}{2^{n}}\right)\left(\sum_{i=1}^{n-1} \frac{1}{2^{i}}+\sum_{i=n}^{\infty} \frac{1}{4^{i}\|f\|}\right) \leq\left(1+\frac{1}{2^{n}}\right)\left(\sum_{i=1}^{n-1} \frac{1}{2^{i}}+\sum_{i=n}^{\infty} \frac{1}{4^{i}}\right) \\
& \leq\left(1+\frac{1}{2^{n}}\right)\left(1-\frac{1}{2^{n-1}}+\frac{1}{3 \cdot 4^{n-1}}\right)<\left(1+\frac{1}{2^{n}}\right)\left(1-\frac{1}{2^{n}}\right)<1 .
\end{aligned}
$$

Consequently, $\left|f(x) h_{0}(x)\right|<1$ for every $x \notin E\left(h_{0}\right)$, as claimed. 
To prove the "moreover" part, let $E$ be a peak set for $A$ containing $x_{0}$, and let $h_{*} \in A$ be such that $E=E\left(h_{*}\right)$. Then (7) holds for the function $h_{0} h_{*}$, and $E\left(h_{0} h_{*}\right) \subset E$ since $\left|h_{*}(x)\right|<1$ on $X \backslash E$.

Corollary 3.2. Let $A$ be as in Proposition 3.1, $x_{0} \in \mathcal{P}(A)$ and $f\left(x_{0}\right) \neq 0$ for some $f \in A$. If $h_{0} \in \mathcal{P}_{x_{0}}(A)$ satisfies equality (7), then $\inf _{h \in \mathcal{F}_{x_{0}}(A)}\|f h\|=\left\|f h_{0}\right\|=\left|f\left(x_{0}\right)\right|$.

Proof. Let $x_{0} \in p(A)$. Proposition 3.1 (The Strong Multiplicative Bishop's Lemma) yields

$$
\|f h\|=\sup _{\xi \in X}|f(\xi) h(\xi)| \geq\left|f\left(x_{0}\right) h\left(x_{0}\right)\right|=\left|f\left(x_{0}\right) h_{0}\left(x_{0}\right)\right|=\max _{\xi \in X}\left|f(\xi) h_{0}(\xi)\right|=\left\|f h_{0}\right\|
$$

for any $h \in \mathcal{F}_{x_{0}}(A)$. According to Lemma 2.2. $\left\|f h_{0}\right\|=\inf _{h \in \mathcal{F}_{x_{0}}(A)}\|f h\|=\left|f\left(x_{0}\right)\right|$.

The next theorem extends the main results of [1, 2, 7, 8, 10, 11, 12] on algebra isomorphisms.

THEOREM 3.3. Let $X$ be a locally compact Hausdorff space and $A \subset C(X)$ be a dense subalgebra of a function algebra without unit, such that $X=\partial A$ and $p(A)=\delta A$. If a surjection $T: A \rightarrow B$ onto a function algebra $B$ without unit is weakly peripherallymultiplicative, i.e. $\sigma_{\pi}(T f T g) \cap \sigma_{\pi}(f g) \neq \emptyset$ for all $f, g \in A$, and preserves the peripheral spectra of algebra elements, i.e. $\sigma_{\pi}(T f)=\sigma_{\pi}(f), f \in A$, then $T$ is a bijective $\psi$-composition operator on $p(B)$, i.e. $(T f)(y)=f(\psi(y))$ for all $f \in A$ and $y \in p(B)$, with respect to a homeomorphism $\psi: p(B) \rightarrow p(A)$. In particular, $A$ is necessarily a function algebra and $T$ is an algebra isomorphism.

Proof. Let $y_{0} \in p(B)=\delta B$. The condition $\sigma_{\pi}(T f T g) \cap \sigma_{\pi}(f g) \neq \emptyset$ implies that $T$ is sup-norm-multiplicative. i.e. $\|T f T g\|=\|f g\|$ for every $f, g \in A$. If $\psi: p(B) \rightarrow p(A)$ is the homeomorphism from Theorem 2.9, then $|(T f)(y)|=|f(\psi(y))|$ for all $y \in p(B)$ and $f \in A$. Clearly, $(T f)(y)=f(\psi(y))$ whenever $(T f)(y)=0$. If $(T f)\left(y_{0}\right) \neq 0$, let $V \subset Y$ be an open neighborhood of $y_{0}$. By Proposition 3.1 (The Strong Multiplicative Bishop's Lemma) there exists a $k \in \mathcal{P}_{y_{0}}(B)$ such that $E(T f \cdot k)=E(k) \subset V$ and $\sigma_{\pi}(T f \cdot k)=\left\{(T f)\left(y_{0}\right)\right\}$. Note that if $h \in T^{-1}(k)$ then $(T f)\left(y_{0}\right) \in \sigma_{\pi}(f h)$ since, by the hypotheses, $\sigma_{\pi}(T f \cdot k) \cap \sigma_{\pi}(f h) \neq \emptyset$. Therefore, there is a point $x_{1} \in p(A)$ so that $(T f \cdot k)\left(y_{0}\right)=(f h)\left(x_{1}\right)$. Since $\psi$ is surjective, there is an $y_{1} \in p(B)$ so that $x_{1}=\psi\left(y_{1}\right)$. Hence,

$$
(T f)\left(y_{0}\right)=(T f)\left(y_{0}\right) \cdot k\left(y_{0}\right)=f\left(\psi\left(y_{1}\right)\right) \cdot h\left(\psi\left(y_{1}\right)\right),
$$

and $\left|(T f)\left(y_{0}\right)\right|=\left|(T f)\left(y_{0}\right)\right|\left|k\left(y_{0}\right)\right|=\left|f\left(\psi\left(y_{1}\right)\right)\right|\left|h\left(\psi\left(y_{1}\right)\right)\right|=\left|(T f)\left(y_{1}\right)\right|\left|k\left(y_{1}\right)\right|$, by Theorem 2.9 and since $\left\{(T f)\left(y_{0}\right)\right\}=\sigma_{\pi}(T f \cdot k)$. Hence $y_{1} \in E(T f \cdot k)=E(k) \subset V$ by the choice of $k$. Thus $k \in \mathcal{F}_{y_{1}}(B)$, and therefore, $\left|h\left(\psi\left(y_{1}\right)\right)\right|=\left|k\left(y_{1}\right)\right|=1$, by Theorem 2.9 . Since, by the hypotheses, $\sigma_{\pi}(h)=\sigma_{\pi}(k)=\{1\}$, it follows that $h\left(\psi\left(y_{1}\right)\right)=1$. Now equality (8) becomes $(T f)\left(y_{0}\right)=f\left(\psi\left(y_{1}\right)\right)$. Hence any neighborhood $V$ of $y_{0}$ contains a point $y_{1} \in p(B)$ such that $(T f)\left(y_{0}\right)=f\left(\psi\left(y_{1}\right)\right)$. The continuity of $f, T f$ and $\psi$ implies that $(T f)\left(y_{0}\right)=f\left(\psi\left(y_{0}\right)\right)$.

In particular, Theorem 3.3 holds for operators between two function algebras without units. Since, as a $\psi$-composition operator on $p(B), T$ is a sup-norm isometric algebra isomorphism, Theorem 3.3 implies the following necessary and sufficient conditions for operators between two algebras of functions to be linear and multiplicative: 
TheOREm 3.4. Let $X$ be a locally compact Hausdorff space and $A \subset C(X)$ be a dense subalgebra of a function algebra without unit, such that $X=\partial A$ and $p(A)=\delta A$. $A$ surjection $T: A \rightarrow B$ onto a function algebra $B$ without unit is a sup-norm isometric algebra isomorphism if and only if $T$ is weakly peripherally-multiplicative and preserves the peripheral spectra of algebra elements.

Note that the operator $T$ in Theorem 3.4 is not assumed a priori to be linear, multiplicative, or continuous. Since a multiplicative operator that preserves the peripheral spectra is automatically weakly peripherally-multiplicative, Theorem 3.4 implies:

Corollary 3.5. Let $X$ be a locally compact Hausdorff space and $A \subset C(X)$ is a dense subalgebra of a function algebra without unit, such that $X=\partial A$ and $p(A)=\delta A$. $A$ multiplicative surjection $T: A \rightarrow B$ onto a function algebra $B$ without unit is a sup-norm isometric algebra isomorphism if and only if $T$ preserves the peripheral spectra of algebra elements.

If the algebras have units, then, similarly to [8, Corollary 3], we obtain:

COROLlary 3.6. Let $X$ be a locally compact Hausdorff space, $A \subset C(X)$ be a unital dense subalgebra of a uniform algebra such that $X=\partial A$ and $p(A)=\delta A$, and let $B$ be a uniform algebra. If $T: A \rightarrow B$ is a peripherally-multiplicative surjection, i.e.

$$
\sigma_{\pi}(T f T g)=\sigma_{\pi}(f g)
$$

for all $f, g \in A$, such that $T 1 \in B^{-1}$, then there is a homeomorphism $\psi: p(B) \rightarrow p(A)$ and $a \kappa \in B$ with $\kappa(y)= \pm 1$ for all $y \in p(B)$, so that $T$ is a $\kappa$-weighted $\psi$-composition operator on $p(B)$, i.e. $(T f)(y)=\kappa(y) f(\psi(y))$ for all $f \in A$ and $y \in p(B)$. In particular, $T$ is linear and $A$ is a uniform algebra.

Proof. Equality 9 implies that $T$ is norm-multiplicative and $\sigma_{\pi}\left((T 1)^{2}\right)=\sigma_{\pi}(1)=\{1\}$. Since, by Theorem $2.9|T 1|=1$ we deduce that $(T 1)^{2}=1$, and therefore, $(T 1)(y)= \pm 1$ for all $y \in p(B)$. The operator $S: A \rightarrow B$ defined as $S f=T 1 T f$ is surjective, and $\sigma_{\pi}(S f S g)=\sigma_{\pi}\left((T 1)^{2} T f T g\right)=\sigma_{\pi}(T f T g)=\sigma_{\pi}(f g)$. Therefore, $S$ satisfies the hypotheses of Theorem 3.4 and hence $(T 1 T f)(y)=(S f)(y)=f(\psi(y))$ for some homeomorphism $\psi: \delta B \rightarrow p(A)$. Therefore, $(T f)(y)=(T 1)^{-1}(y) f(\psi(y))=\kappa(y) f(\psi(y))$ for all $f \in A$ and $y \in p(B)$, where $\kappa=(T 1)^{-1}$.

Acknowledgements. This paper extends and generalizes results presented at the $19^{\text {th }}$ International Conference on Banach Algebras held at Będlewo, July 14-24, 2009. The support for the meeting by the Polish Academy of Sciences, the European Science Foundation under the ESF-EMS-ERCOM partnership, and the Faculty of Mathematics and Computer Science of the Adam Mickiewicz University at Poznań is gratefully acknowledged.

Thanks are due also to F. Sady for the helpful remarks and comments, and to the referee for the valuable suggestions, which significantly improved the exposition.

\section{References}

[1] S. Grigoryan and T. Tonev, Shift-Invariant Uniform Algebras on Groups, Monografie Matematyczne 68, New Series, Birkhäuser Verlag, Basel-Boston-Berlin, 2006. 
[2] O. Hatori, T. Miura and H. Takagi, Characterization of isometric isomorphisms between uniform algebras via non-linear range-preserving properties, Proc. Amer. Math. Soc. 134 (2006), 2923-2930.

[3] O. Hatori, T. Miura and H. Takagi, Unital and multiplicatively spectrum-preserving surjections between semi-simple commutative Banach algebras are linear and multiplicative, J. Math. Anal. Appl. 326 (2007), 281-296.

[4] M. Hosseini and F. Sady, Multiplicatively range-preserving maps between Banach function algebras, J. Math. Anal. Appl. 357 (2009), 314-322.

[5] M. Hosseini and F. Sady, Multiplicatively and non-symmetric multiplicatively norm-preserving maps, Cent. Eur. J. Math., to appear.

[6] A. Jimenez-Vargas, A. Luttman and M. Villegas-Vallecillos, Weakly peripherally multiplicative surjections of pointed Lipschitz algebras, Rocky Mountain J. Math., in print.

[7] S. Lambert, A. Luttman, and T. Tonev, Weakly peripherally-multiplicative operators between uniform algebras, in: Contemp. Math. 435, Amer. Math. Soc., 2007, 265-281.

[8] A. Luttman and T. Tonev, Uniform algebra isomorphisms and peripheral multiplicativity, Proc. Amer. Math. Soc. 135 (2007), 3589-3598.

[9] L. Molnár, Selected Preserver Problems on Algebraic Structures of Linear Operators and on Function Spaces, Lecture Notes in Mathematics 1895, Springer-Verlag, Berlin, 2007.

[10] L. Molnár, Some characterizations of the automorphisms of $B(H)$ and $C(X)$, Proc. Amer. Math. Soc. 130 (2001), 111-120.

[11] N. V. Rao and A. K. Roy, Multiplicatively spectrum-preserving maps of function algebras, Proc. Amer. Math. Soc. 133 (2005), 1135-1142.

[12] N. V. Rao and A. K. Roy, Multiplicatively spectrum-preserving maps of function algebras II, Proc. Edinb. Math. Soc. (2) 48 (2005), 219-229.

[13] R. Yates, Norm-preserving criteria for uniform algebra isomorphism, Ph.D. Dissertation, The University of Montana, Missoula, 2009.

[14] W. Żelazko, Banach Algebras, Elsevier, 1973. 
